\title{
Nicotine and symptoms of schizophrenia: the 2017 picture
}

\author{
Author: \\ Jacqueline Conway \\ E-mail: jackie.conway@doctors.org.uk
}

\begin{abstract}
The tendency of people who suffer with schizophrenia to consume large amounts of cigarettes and to extract more nicotine from these cigarettes is well-known. A sizeable body of work investigating this has concluded that smoking in people with schizophrenia improves the dysfunctional sensory gating intrinsic to this disease. This has been recognised at least since the late 1980s. Consequently, the pro-cognitive effect of nicotine is now well-recognised and has led to the investigation of nicotinic receptor modulators as potential treatments for schizophrenia. Particular interest has focused on the treatment of negative symptoms in this illness. While there is a solid theoretical basis for this line of drug development, in practice, later-stage drug trials of compounds such as TC-5619 (bradanicline) in Phase 2 and EVP-6124 (encenicline) in Phase 3 have been disappointing. Numerous intolerable side-effects have been important in limiting the clinical use of such compounds. It is conceivable that the reason for intolerable side-effects might be a consequence of the wide distribution of nicotinic cholinergic receptors: $\alpha_{7}$ nicotinic receptors are found in several cerebral areas, in the spleen and in lymphocytes at lymph nodes.

Later-stage drug development trials which reveal insurmountable administration problems are perhaps indicative of the great practical distance between theoretical recognition of a potential therapeutic agent and its eventual translational appearance in the armory against an illness. Given that this is the case it may still be worthwhile to look at the simple administration of exogenous nicotine via e-cigarettes or chewing-gum as an adjunctive agent in the treatment of schizophrenia in the interim. More prosaically, the banning of smoking in many hospital premises in the UK has led to a difficulty in the practical management of acutely ill people with schizophrenia in the in-patient setting. In a group of people who are profoundly psychiatrically unwell, and often physically compromised to some degree because of their cigarette consumption, alternative management techniques for the latter must be explored. Use of exogenous nicotine replacement in a less harmful delivery manner than cigarettes in a group of people who have a clear physiological reason for smoking -i.e. to increase their deficient endogenous cerebral nicotine levels - is worthwhile to investigate. Very recent work in exactly this direction has been undertaken. Nursing management of such patients may be greatly improved as a result, as may their on-going engagement with the therapeutic team. Here is an illustration of how theoretical considerations eventually come to have a pragmatic consequence in the day-to-day management of people with severe and enduring mental illnesses like schizophrenia.
\end{abstract}




\section{Background: the neuropathology of schizophrenia}

The increased tendency of psychiatric in-patients to smoke is well-known. Explanations for this used to focus on the ward lifestyle. Even as recently as 2006, the association between admission with schizophrenia, smoking and high dose of antipsychotic medication was being reported in Finland (Salongkas et al 2006). When associations such as this are consistently found, it is imperative to investigate the reasons for the association. It must always be remembered that correlation is not the same as causality. While a correlation gives a clue to the possible causal mechanism, it is not the full explanation. It has consistently been found that habitual smoking is far more pronounced in people suffering from schizophrenia: 76 to $88 \%$ of schizophrenic patients (e.g. Kelly et al 1999, Patkar et al 2002). This topic began to be deliberated upon in the 1970s and 1980s. Another relevant aspect was eventually found to be the fact that the aromatic hydrocarbons in cigarette smoke induce catabolic cytochrome enzymes (Zevin and Benowitz 1999, Desai et al 2001). As such, heavy cigarette smokers - those who smoke more than ten cigarettes per day - require higher doses of psychotropic drugs. People who smoke at this level sustain nicotine levels (as measured by serum levels of its metabolite, cotinine) of $5 \mathrm{ng} / \mathrm{ml}$ trough up to $35 \mathrm{ng} / \mathrm{ml}$ peak (Hong et al 2011). In non-smokers, serum cotinine levels approximate $3 \mathrm{ng} / \mathrm{ml}$ (Benowitz et al 2009). Therefore, those who smoke more than ten cigarettes per day have a consistently higher circulating nicotine level. In people who suffer from schizophrenia, it is worthwhile to examine the reasons for their increased cigarette consumption. The higher circulating nicotine level affords a clue to the explanation.

When first-generation antipsychotic drugs were commonly used in the in-patient environment, it was reasonable to believe that part of the reason for the heavy consumption of tobacco cigarettes was the drive to lower the level of serum antipsychotic to decrease the occurrence of extra-pyramidal side effects. The hepatic catabolic process for psychotropic drugs has been further detailed and confirmed. As has been stated above, constituents of cigarette smoke- namely, the aromatic hydrocarbons induce the hepatic cytochrome enzymes which break down several drugs and toxins, and this is completely independent of the nicotine component of the cigarette (Benowitz 1999, Desai et al 2001). Tobacco cigarette consumption therefore needs to be taken into account when prescribing for a person who has schizophrenia. The secondgeneration, atypical antipsychotic medications have far less incidence of extrapyramidal side effects but the incidence of smoking in people with schizophrenia remains markedly raised: the figure remains up to eighty-eight per cent, as has been mentioned above. It was therefore clear that the reasons for smoking in schizophrenia are not simply due to in-patient lifestyle, for example. McEvoy and Brown (1999), amongst others, have suggested that smoking is a marker for the more severe forms of schizophrenia. A tremendous body of work has become established on the topic of smoking, nicotine and schizophrenia and has been investigated via numerous methods.

At this point, the role of the tobacco industry must be mentioned. In 2008, Prochaska et al looked at the influence of the companies who sell tobacco in the USA on tobacco use policies in healthcare there. They found that these companies actively promoted the susceptibility of people with schizophrenia to smoke and actively worked against smoking bans in hospital setting in the USA. Prochaska et al examined many documents which were previously secret to uncover the actions of these companies. These corporate actions are clearly unfortunate: the single-minded pursuit of 
financial advantage by the businesses concerned has thrown some unnecessary confusion into a valid body of work that investigates the neuropathology of schizophrenia.

\section{Sensory gating in schizophrenia}

Electrophysiological changes in people who suffer from schizophrenia have been demonstrated to be a useful method of investigating the neurophysiological mechanisms involved.

A robust finding in schizophrenia is the lack of pre-pulse inhibition. Pre-pulse inhibition is an electrophysiological investigation of sensory gating: this refers to electrophysiological measurement of cerebral activity when sensory stimuli are presented in rapid succession. There are numerous articles on this topic, such as Braff et al (2001) which describe the investigatory process. In the well-known two-click paradigm, a stimulus noise is presented against a background of white noise: the stimulus is generally a loud click. The cerebral response to this sensory stimulus is measured by electroencephalography. If a second clicking noise is presented soon after - for example 50 milliseconds or 300 milliseconds later, the evoked response potential at the cerebral vertex is found to be of a lower amplitude both in people who suffer from schizophrenia and in their firstdegree relatives. This is described as P50 or P300 pre-pulse inhibition. This is said to be indicative of sensory gating: cerebral activity is maintained towards the first stimulus until neurological processing of it has been completed. Potter et al (2006) also describe sensory gating as "...the pre-attentional habituation of responses to repeated exposure to the same sensory stimulus." The fact that both people who suffer from schizophrenia and their first-degree relatives show a lack of pre-pulse inhibition demonstrates a possible liability to this illness. However, additional hippocampal pathology is necessary before the potential sensory gating deficit becomes manifest in a psychotic state (Dalack et al 1998, Adler et al1998). It is also probable that the hippocampal pathology must be longstanding. It is well-recognised both clinically and in the research setting, that in firstepisode patients, a longer duration of untreated psychosis is associated with a far less favorable prognosis. This is most probably due to the establishment and reinforcement of dysfunctional cerebral circuitry. Attentional deficits are also found in sustained attention in schizophrenia. Hong et al 2011) investigated sustained visual attention here and found a "...trait-like deficit...across early and chronic states of the disease in medicated and never medicated individuals". This group of researchers noted that exogenous nicotine can also transiently improve sustained attention in people with schizophrenia. This confirms the work of Adler et al in 1998, who investigated $\mathrm{P}_{300}$ pre-pulse inhibition. The deficit in sensory gating, i.e. the loss of pre-pulse inhibition, has been traced to reduced action at $\alpha_{7}$ nicotinic cholinergic receptors: here, exogenously-administered nicotine could only transiently restore prepulse inhibition for up to thirty minutes and the improvement could not be sustained. This is most probably because the neural receptor involved, the $\alpha_{7}$ nicotinic receptor, is one which desensitises quickly. 


\section{The role of the $\alpha_{7}$ nicotinic cholinergic receptor}

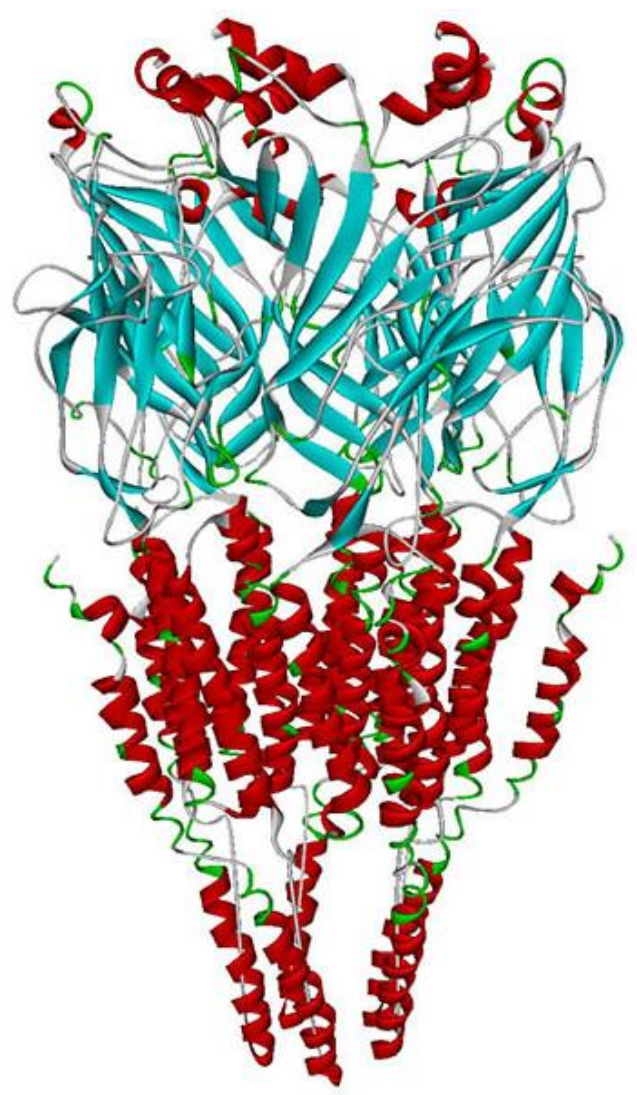

Figure 1. Molecular model of the $\alpha_{7}$ nicotinic cholinergic receptor

Public Domain, https://commons. wikimedia.org/w/index.php?curid=6916339

The molecular model illustrated here permits visualisation of the pentameric receptor structure, which here comprises five homogenous $\alpha_{7}$ molecules. Other nicotinic receptors are heterogeneous, such as the structure, which contains two types of constituent molecule: $\alpha_{4}$ and $\beta_{2}$ : this receptor has three $\beta_{2}$ and two $\alpha_{4}$ molecules. $\alpha_{4} \beta_{2}$ receptors are involved in cerebral mesolimbic projections. They modulate behavioural reinforcement and so are operative in the addictive properties of nicotine and thus of cigarettes. Cell receptors which contain the $\alpha$ subunits are of lower affinity: this is due to their molecular physical configuration. Receptors contacting the $\beta$ subunits are, conversely, high-affinity, again for similar reasons (Mackowick at al 2013). In schizophrenia, the most relevant receptor type is the $\alpha_{7}$ receptor at hippocampal interneurones in area CA 3 (the area designated 3 in the hippocampal Cornis Ammonis). A receptor which contains only $\alpha$ subtypes will therefore have a very low affinity and require a large amount of ligand to activate it. All nicotinic receptor subtypes are ligand-gated ion channels: the ligand is nicotine and the ion is the bivalent calcium $\mathrm{Ca}^{2+}$ ion. It is probable that the binding of nicotine to this cholinergic receptor / the passage of ions through it is achieved via a stereochemical molecular change, i.e. allosteric modulation. Certainly, allosteric modulation at the $\alpha_{7}$ receptor is one of the pharmacological avenues being explored in the on-going investigations of new pharmacological treatments for schizophrenia (Timmerman et al 2007). $\alpha_{7}$ 
receptors operate in correctly-functioning sensory gating, e.g. allowing habituation to repetitive sensory stimuli. In the healthy functional state, trivial stimuli are disregarded. Where sensory gating does not function correctly, these trivial stimuli can acquire inappropriate, even delusional significance because they can no longer be screened out. Delusions can be understood as the conscious interpretation of dysfunctional neurophysiological activity.

At interneurones in the hippocampal CA3 region, sufficient nicotine allows the $\alpha_{7}$ receptor to permit influx of calcium into these cells, so depolarising them. These are inhibitory neurones - therefore, sufficient nicotine allows the correct level of inhibition to occur. In this way, repeated sensory stimuli are not sent for further cortical processing, but are disregarded. Similarly, if multiple stimuli are presented, they are prevented from being further developed, i.e. into conscious sensory perception, until the management of the initial stimulus is completed. This is the basis of sensory gating, which can only function correctly if there is a sufficient level of cerebral nicotine. This is usually endogenous, but can be supplemented from external sources, such as tobacco cigarette smoking. As stated above, the $\alpha_{7}$ receptor is of low affinity, thus a high level of cerebral nicotine is required. People who suffer from schizophrenia are thus supplementing their deficient endogenous nicotine levels with a high level of tobacco cigarette consumption. Nicotine readily crosses the blood-brain barrier, making cigarette smoking an efficient way of increasing cerebral nicotine levels. Of course, the negative effects to a person's physical health as a result of tobacco cigarette smoking are notorious.

The $\alpha_{7}$ nicotinic receptor is a problematic one: as well as having a low affinity for nicotine it desensitises rapidly (Dalack et al 1998). This, therefore, is why nicotine replacement therapy (NRT) with slow-release nicotine patches is unsuitable as an adjunctive treatment for schizophrenia: the hippocampal $\alpha_{7}$ receptor would become desensitised and the desired effect improved sensory gating - is not achieved. A more suitable mode of NRT in schizophrenia is one which replicates the short burst of increased circulating nicotine provided by cigarette smoking. Nicotine chewing-gum or - currently (2017) controversially - the use of an e-cigarette are the two clearest candidates for this.

\section{Excitotoxic cell damage in schizo- phrenia}

At the hippocampus, if cholinergic stimulation is not sufficient, new memory is formed and there is a continual alteration of auto-associative memory. Therefore, in a state of low cholinergic stimulation, the cerebral cortex is bombarded with information which takes on an inappropriate personal significance (Adler et al 1998). This constant over-stimulation leads to excitoxicity (Jarskog 2006) and, eventually, to cerebral tissue loss. Where there is constant depolarization of involved neurones, their cell content eventually becomes depleted. As the involved neurones cannot replace their constituents at a sufficient rate, their cell bodies become redundant. The non-functional neurone is cleared by cerebral microglia. Microglial action is exactly analogous to macrophage activity in the peripheral circulation. This, then, is the basis of cortical tissue loss in chronic schizophrenia. - The cerebral atrophy which used to be seen at post mortem examination of the brains of people suffering from chronic schizophrenia is readily explained by this mechanism. A recent Turkish study (Beyazyüz et al 2016) found that there were increased levels of serum markers of apoptosis in people with deficit state schizophrenia: what used to be 
pejoratively termed 'burnt-out' schizophrenia. Beyazyüz et al investigated apoptosis by measuring serum nucleosome levels. Nucleosomes are sites within a cell where DNA is degraded as part of programmed cell death, apoptosis. Therefore, a higher serum nucleosome level is indicative of increased apoptosis. Beyazyüz et al compared serum from people with deficit state schizophrenia and nondeficit state schizophrenia. People with deficit state schizophrenia were found to have significantly increased apoptotic activity, which relates to the marked negative symptoms predominant in this type of schizophrenia. Tissue loss has been found in previous post-mortem studies to be especially apparent in the pre-frontal cortex. Indeed, hypo-frontality is one of the neuropsychiatric features of chronic schizophrenia. Hypo-frontality comprises many of the features evident as negative symptoms of schizophrenia, such as anhedonia, bradykinesia, poverty of movement, speech and thought. These symptoms are indicative of a lack of initiation of action in each of these domains. Loss of cerebral cortex in the frontal lobes is certainly one explanation for negative symptoms in schizophrenia, and perhaps explains why these symptoms can be so difficult to treat. An important view here (Lieberman 1999) is that schizophrenia should be regarded as a neurodegenerative disease because of the serious risk of cerebral tissue loss caused by excitotoxicity. Lieberman believes that the re-designation of schizophrenia as a potential neurodegenerative disease will change the view both of those who suffer with this illness and those who treat it. Perhaps, in the former case, this will aid compliance with therapeutic measures. The aim of treatment with nicotinic receptor modulators can therefore be an attempt to prevent the neurodegenerative changes of schizophrenia. The same could be said of all modes of antipsychotic treatment used in this illness, but the direct prevention of excitotoxic cell damage via, e.g. allosteric modulation at the $\alpha_{7}$ nicotine receptor (Timmerman et al 2007) is an especially appropriate understanding of antipsychotic treatment. Therefore, more recent work extends the earlier work of Lieberman and Jarskog: if excitotoxicity can be prevented, there is no progression to the neurodegenerative condition of deficit state schizophrenia.

\section{Recent drug developments}

Walling et al (2016) report on a Phase 2 drug trial of TC 5619 (bradanicline), an $\alpha_{7}$ nicotinic receptor agonist being developed as a new treatment for schizophrenia. This group of workers again refers to the copious available evidence supporting this type of drug development. Following on from the discussion above, outcome measurements included assessment of negative symptoms as one of the outcome measures. Their study was an international, multicentre project: it did not find any benefit when TC 5619 was used in terms of negative symptom profile, functional measures or cognitive measures.

However, it was found that schizophrenic subjects who were also tobacco users showed more beneficial results than non-tobacco users. Walling et al hypothesise that this is because this group received a two-fold improvement to their nicotinic transmission. Their explanation is that TC-5619 acts pre-synaptically and they theorise that presynaptic action at $\alpha_{4} \beta_{2}$ receptors is pro-cognitive. Their conclusion differs slightly from other workers in this field. The more general consensus is that $\alpha_{7}$ nicotinic receptor action is beneficial and pro-cognitive, while $\alpha_{4} \beta_{2}$ receptor action has a greater role in reinforcement of action and, potentially, in addiction.

Later stage investigation of other new anti-schizophrenia drugs in development has 
found that cognitive-enhancing effects are were not consistently seen in people with schizophrenia in Phase 2 trials (Wallace and Bertrand 2013). Newer research molecules, when tested in Phase 1 clinical trials demonstrated the cognitive enhancement expected from the theoretical foundations of this work. Unfortunately, Phase 1 trials of these molecules had to be abandoned due to intolerable cardiovascular side-effects. The exact nature of these were not specified in Wallace and Bertrand's review, but vagal nerve involvement may have been relevant, as implied by de Jonge and Ulloa in 2007.

\section{The $\alpha_{7}$ nicotinic receptor and inflammation}

Investigation into gastrointestinal pathology in the Netherlands (de Jonge and Ulloa 2007) has revealed the importance of cholinergic reciprocal function in the mediation of inflammation. This group noted that previous workers had found that the inflammation inherent in conditions such as ulcerative colitis worsened when the affected individuals stopped smoking. They further investigated this process and discovered the profound anti-inflammatory effect of nicotine when acting at $\alpha_{7}$ nicotinic acetylcholine receptors ( $\left.\alpha_{7} \mathrm{NACHRs}\right)$. With reference to the central nervous system, it has been found that the $\alpha_{7}$ nicotinic receptors on cerebral microglia act as antiinflammatory mediators. As stated above, microglia are the cerebral cells which are analogous to macrophages in the peripheral circulation and so are therefore operative in processes such as tissue loss via excitotoxicity. Sufficient circulating nicotine down-regulates microglial activity, thus has a direct effect on the apoptotic pathway mentioned earlier. This effect is especially marked in those regions where the $\alpha_{7} \mathrm{NACHR}$ is expressed in greater numbers, such as in the hippocampus and the cerebral cortex. Therefore, nicotine can be neuroprotective and ultimately enhance cognitive function (de Jonge and Ulloa 2007).

\section{Clinical implications}

Finally, an Italian research group has reported on the use of electronic cigarettes in people with schizophrenia who were smokers and who had been admitted to an in-patient facility. The subjects were followed up for one year, which is a welcome contrast to other studies which stop after the markedly insufficient period of twelve weeks. Caponnetto et al found that $50 \%$ of their study subjects achieved sustained abstinence from tobacco consumption. They also quantified symptoms in their subjects with the wellknown and consistently validated instruments SANS (Scale for the Assessment of Negative Symptoms) and SAPS (Scale for the Assessment of Positive Symptoms). Although they mention that there were no negative findings on either scale measurement, they unfortunately do not elaborate on this data. This is most probably because their focus was on continued tobacco use or otherwise. However, their work undoubtedly demonstrates that ecigarettes can be used as a physical healthpromoting tool for people who do not want to stop smoking such as those with schizophrenic illness. It is worthwhile to extend their work and to investigate whether e-cigarettes can be used as adjunctive agents in symptomatic schizophrenia. As stated above here - as well as by several others there is a possibility of symptomatic relief in schizophrenia by enhancing hippocampal sensory gating. The other, very beneficial aspect of electronic cigarette use is that the previous smoker's respiratory health is greatly improved by no longer inhaling toxic aromatic hydrocarbons. If that smoker is a person with schizophrenia, then cessation of intake of inhaled toxic aromatic 
hydrocarbons means that any antipsychotic medication that they consume will be no longer be so aggressively catabolised by liver enzymes. Thus, there could be a double-edged reason for eventual lower medication requirement: fewer symptoms and reduced hepatic catabolism. People with schizophrenia are known to have an increased incidence of respiratory and cardiovascular disease (Partti et al 2015). Quite simply, if they are no longer smoking as many cigarettes, their respiratory health can only be improved as a result (ibid.).

As we are at a very early stage in electronic cigarette use, it is the ideal time to further investigate these as potential therapeutic tools. It is also possible at this early stage to regulate the components of ecigarettes so that potential harmful solvent additives can be excluded from further manufacture. In conclusion, it is useful to recall the difficulties of management of a person who is acutely unwell with schizophrenia. If the overwhelming weight of evidence leads to the clinical use of electronic cigarettes as an adjunctive measure in the management of schizophrenia, the potential beneficial effects could be considerable. By allowing measures such as use of electronic cigarettes in the in-patient environment, it is quite possible that the number of disruptive incidents on the ward would be decreased. In-patients would therefore be far less affected by the smoking ban present in UK NHS hospitals because they are using a mode of nicotine replacement which could allow them to experience an in-patient stay less negatively than at present. A longerterm consequence may be improved engagement with the therapeutic team and, thus, improved compliance with antipsychotic medication. A consequence of this latter situation therefore is that the progression to the neurodegenerative deficit state may be avoided by the adoption of this mode of adjunctive treatment: hence there is another potential patient benefit. 


\section{References}

Lawrence E. Adler, Ann Olincy, Merilyne Waldo, Josette G. Harris, Jay Griffith. Karen Stevens, Karen Flach, Herbert Nagamoto, Paula Bickford, Sherry Leonard and Robert Freedman. Schizophrenia, Sensory Gating and Nicotinic Receptors. Schizophrenia Bulletin 1998 24(2): 189-202

Murat Beyazüz, Tarkan Küfeciler, Leyla Bulut, Cüneyt Ünsal, Yakup Alabyrak, Esra Soydas Akyol, Saliha Baykal, Murat Kuloglu, Kenju Hashimoto. Increased serum levels of apoptosis in deficit syndrome schizophrenia patients: a preliminary study Neuropsychiatric Disease and Treatment 2016: 12 1261-1268

Neal L. Benowitz, John T. Bernert, Ralph S. Caraballo, David B. Holliday and Jiantong Wang Optimal Serum Cotinine Levels for Distinguishing Cigarette Smokers and Nonsmokers Within Different Racial/Ethnic Groups in the United States Between 1999 and 2004 American Journal of Epidemiology 2008:169: 236-248

David L. Braff, Mark A. Geyer, Gregory A. Light, Joyce Sprock, William Perry, Kirstin S. Cadenhead, Neal R. Swerdlow et al. Impact of prepulse characteristics on the detection of sensorimotor gating deficits in schizophrenia Schizophrenia Research 2001 49: 1-2 171-178

Pasquale Caponnetto, Roberta Auditore, Cristina Russo, Giorgio Carlo Cappello and Riccardo Polosa Impact of an Electronic Cigarette on Smoking Reduction and Cessation in Schizophrenia Smokers: A Prospective 12-Month Pilot Study. International Journal of Environmental Research and Public Health 2013, 10, 446461.

Copyright 2017 KEI Journals. All Rights Reserved
Gregory W. Dalack, Daniel J. Healy and James H. Meador-Woodruff. Nicotine Dependence in Schizophrenia: Clinical Phenomena and Laboratory Findings. American Journal of Psychiatry 1998; 155(11): 1490-1501

Michael C. Davis, William P. Horan, Stephen R. Marder. Psychopharmacology of the negative symptoms: Current status and prospects for progress. European Neuropsychopharmacology 2013: 1-11. http://dx.doi.org.10.1016/j.euroneuro.2013.1 $\underline{0.010}$

W.J.de Jonge and L. Ulloa. The alpha 7 nicotinic acetylcholine receptor as a pharmacological target for inflammation. British Journal of Pharmacology 2007 151:915-929

Desai HD, Seabolt J, Jann MW. Smoking in patients receiving psychotropic medications: a pharmacokinetic perspective. CNS Drugs 2001; 15(6):469-94

L. Elliott Hong, Matthew Schroeder, Thomas J. Ross, Brittany Buchholz, Betty Jo Salmeron, Ikwunga Wonodi, Gunvant K. Thaker and Elliot A. Stein. Nicotine Enhances but Does Not Normalize Visual Sustained Attention and the Associated Brain Network in Schizophrenia Schizophrenia Bulletin 2011 37(2): 416-425

L. Frederik Jarskog Apoptosis in schizophrenia: pathophysiologic and therapeutic considerations. Current Opinion in Psychiatry 19:307-312, 2006

Kelly Ciara, McCreadie Robin G., Smoking Habits. Current Symptoms, and Premorbid Characteristics of Schizophrenic Patients in Nithsdale, Scotland. American Journal of Psychiatry1999; 156(11): 1751-1757 
Aaron Kuckinski, Scott Wersinger, Ewa K. Stachowiak, Chani Becker, Pat Lippiello, Merouane Bencherif and Michal K. Stachowiak. The effects of varenicline on sensory gating and exploratory behaviour with pretreatment with nicotinic or 5-HT3A receptor antagonists. Behavioural Pharmacology 2015; 26:217-226

Jeffrey A. Lieberman. Is schizophrenia a neurodegenerative disorder? A clinical and neurobiological perspective. Biological Psychiatry 1999; 46(6):729-739

Kristen A. Mackowick, Mera S. Barr, Victoria C. Wing, Rachel A. Rabin, Clairelaine Ouellet-Plamondon, Tony P. George. Neurocognitive endophenotypes in schizophrenia: Modulation by nicotinic receptor systems. Progress in Neuropharmacology \& Biological Psychiatry 2013 http://dx.doi.org.10.1016/j.pnpbp.2013.07.01 $\underline{0}$

Joseph P McEvoy, Shirley Brown. Smoking in first-episode patients with schizophrenia. American Journal of Psychiatry 1999; 156(7): 1120

Sharon Mexal, Ralph Berger, Judy Logel, Randal G Ross, Robert Freedman and Sherry Leonard Differential Regulation of $\alpha 7$ Nicotinic Receptor Gene (CHRNA7) Expression in Schizophrenic Smokers. Journal of Molecular Neuroscience 2010; 40(1-2): 185-195

Krista Partti, Tuula Vasankari, Merja Kanevisto, Jonna Perälä, Sumuli I. Saami, Pekka Jousilahti, Jouko Lönnqvist and Jaana Suivisaari. Lung function and respiratory diseases in people with psychosis: population-based study. British Journal of Psychiatry 2015207 (1) 37-45; DOI: 10.1192/bjp.bp.113.141937
Patkar Ashwin A., Gopalakrishnan Raman, Lundy Allan, Leon Frank T., Certa Kenneth M., Weinstein Stephen P. Relationship between tobacco smoking and positive and negative symptoms inschizophrenia. Journal of Nervous and Mental Disease 2002; 190 (9): 604-610

Marina R. Piciotto, Yann S. Mineur. Molecules and circuits involved in nicotine addiction: The many faces of smoking. Neuropharmacology 76 (2014) 545-553

David Potter, Ann Summerfelt, James Gold and Robert W Buchanan. Review of Clinical Correlates of P50 Sensory Gating Abnormalities in Patients with SchizophreniaSchizophrenia Bulletin 2006; 32(4): 692-700

Judith J.Prochaska, Sharon M. Hall and Lisa A. Bero. Tobacco Use Among Individuals With Schizophrenia: What Role Has the Tobacco Industry Played? Schizophrenia Bulletin 2008; 34(3): 555-567.

R.K.R. Salongkas, T. Honkonen, E. Stengård, A-M Koivisto, J. Hietala. Cigarette smoking in long-term schizophrenia. European Psychiatry 2006 21: $219-223$

Daniel B.Timmerman, Jens Halvard Grønlien, Kathy L. Kolhaas, Elsebet $\varnothing$. Nielsen, Eva Dam, Tino D. Jørgensen, Philip K. Ahring, Dan Peters, Dorte Holst, Jeppe K Christensen, John Malysz, Clark A. Briggs, Murali Gopalakrishnan and Gunnar M. Olsen. An Allosteric Modulator of the $\alpha 7$ Nicotinic Acetylcholine Receptor Possessing Cognition-Enhancing Properties In Vivo. The Journal of Pharmacology And Experimental Therapeutics 2007; 323:294307 
Medical Research Archives, Vol. 5, Issue 7, July 2017

Nicotine and symptoms of schizophrenia: the 2017 picture

Tanya L. Wallace \& Daniel Bertrand. Alpha 7 neuronal nicotinic receptors as a drug target in schizophrenia. Expert Opnion in Therapeutic Targerts 2013 17(2): 139-155

David Walling, Stephen R. Marder, John Kane, Wolfgang Fleischhacker, Richard S.E. Keefe, David A. Hosford, Chris Dvergsten, Anthony C. Segreti, Jessica S. Beaver, Steven M. Toler, John E. Jett and Geoffrey C. Dunbar. Phase 2 Trial of an Alpha-7 Nicotinic Receptor Agonist (TC-5619) in Negative and Cognitive Symptoms of Schizophrenia. Schizophrenia Bulletin 2016; 42(2): $335-343$
Yang Yang, Constantinos D. Paspalas, Lu E. Jin, Marina Piciotto, Amy F. T. Arnstern and Min Wang. Nicotinic $\alpha 7$ receptors enhance NMDA cognitive circuits in dorsolateral prefrontal cortex. PNAS 2013; www.pnas.org/cgi/doi/10.1073/pnas.130784 $\underline{9110}$

Zevin S, Benowitz NL. Drug interactions with tobacco smoking. An update. Clinical Pharmacokinetics 1999; 36(6): 425-38 